\section{Wobble-based on-chip calibration circuit for temperature independent oscillators}

\author{
V. De Smedt, W.Steyaert, W. Dehaene and G. Gielen
}

An automatic calibration circuit for temperature- and processindependent oscillators and timing circuits is presented. The circuit monitors the dynamic gate-source voltage of a MOS transistor of which the temperature is changing. By adapting the bias current through this transistor towards the zero-temperature-coefficient (ZTC) point of the $\mathrm{V}_{\mathrm{GS}}$, the gate-source voltage can be used in temperature-independent timing circuits. Due to the dynamic nature of this regulation circuit, the technique is insensitive to mismatch and process variations. The presented technique is applied to a low-noise oscillator to make it less temperature sensitive with a factor of 8 .

Introduction: During the last decades, a trend towards fully integrated electronic systems is arising. Also in wireless sensor applications, where tens or hundreds of sensors can be placed in the same room, the use of external components needs to be avoided to reduce the cost of the overall system. Temperature independence as well as supply voltage independence are often important specifications $[1,2]$. By making use of the zero temperature coefficient (ZTC) biasing point of a MOS transistor, a temperature-stable reference voltage and current can be obtained. In this way, many high-performance building blocks can be made more robust. However, nanometer CMOS technologies suffer from process variability and mismatch which makes ZTC biasing impossible at design time. This letter proposes a fully integrated, automatic calibration topology for fully integrated systems. The topology is applied to a relaxation oscillator. Note that no external components and no extra calibration steps during or after production are needed.

ZTC Biasing: As shown in [3] each diode-connected MOS transistor has a ZTC biasing point in which the voltage to current ratio is constant as a function of temperature. Fig. 1 shows the current through a diodeconnected MOS transistor as a function of the gate-source voltage at 3 different temperatures. Apart from the ZTC point, it is clearly visible that the temperature dependency of the current through the transistor is opposite at both sides of the ZTC point. When the transistor is biased exactly in the ZTC point, the current and voltage are independent of the temperature. Note that by comparing the voltage or current change of the transistor to the temperature change, one can easily determine the direction in which the biasing must be adapted to reach the ZTC point.

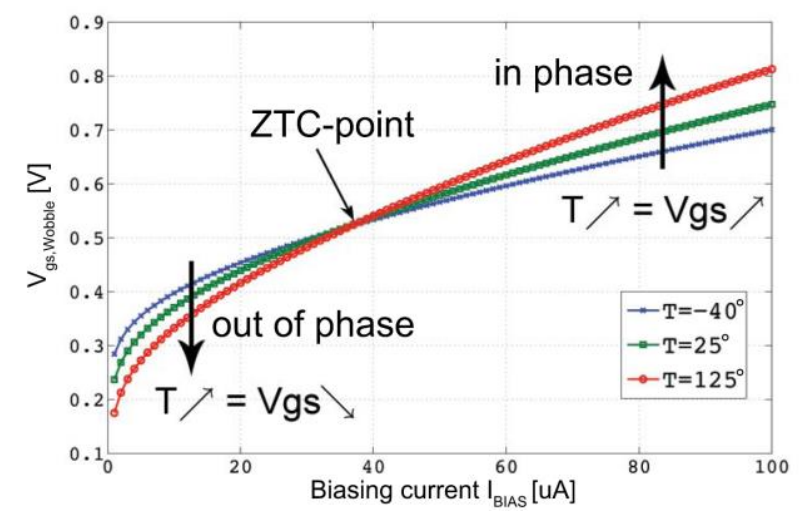

Fig. $1 V_{G S}$ versus $I_{D S}$ plot for a NMOS at different temperatures. The ZTC biasing point is clearly visible. Depending on the biasing point the voltage changes in or out of phase with the temperature.

Dynamic calibration circuit: The calibration circuit consists of 3 main parts: the diode-connected transistor with heating circuit, the phase detector with counter and the current-DAC. A block schematic of the control system is shown in fig. 2. The heater of transistor A is activated periodically. The temperature increase as a function of the input power can be calculated with the formula used in [4]:

$$
Z(\omega, r)=\frac{T(\omega, r)}{P(\omega)}=\frac{1}{2 \pi k r} \exp \left(-r \sqrt{\frac{\omega}{2 D}}\right) \exp \left(-j r \sqrt{\frac{\omega}{2 D}}\right)
$$

where $\mathrm{Z}$ is the thermal impedance between the heater and the point of interest (in this case the diode transistor), $\mathrm{T}$ is the temperature and $\mathrm{P}$ the applied heating power as a function of the pulsation $\omega$ at which the heater is periodically triggered. $\mathrm{D}$ is the thermal diffusivity constant of pure silicon (around $0.88 \mathrm{~cm}^{2} / \mathrm{s}$ at $300 \mathrm{~K}$ [4]), $\mathrm{r}$ the distance between the heater and the point of interest and $\mathrm{k}$ is the thermal conductivity of silicon. Apart from the absolute value of the temperature rise, the formula also shows a phase shift between the input power and the output temperature rise. This phase shift, however, can in this case be neglected because of the small distance between the heater and the transistor of interest. Furthermore, the frequency at which the heater is triggered can be kept low.

Finite element simulations confirm the output of this formula and show that a mean heating power of $10 \mathrm{~mW}$ leads to a temperature variation of $7.3^{\circ} \mathrm{C}$ in the transistor core. As a result of the temperature change, the voltage over the transistor will change either in phase or out of phase with the temperature. The phase difference between the heater's input signal and the resulting gate-source voltage is measured by the phase detector in order to decide either to increase or decrease the biasing current. The current $\mathrm{I}_{2}$ is controlled by a counter, which is incremented or decremented each cycle and drives the current-DAC. In this way, the current 'wobbles' towards the ZTC point. Once the ZTC biasing point is reached, the transistor will behave independently with temperature and the power hungry heating circuit can be switched off.

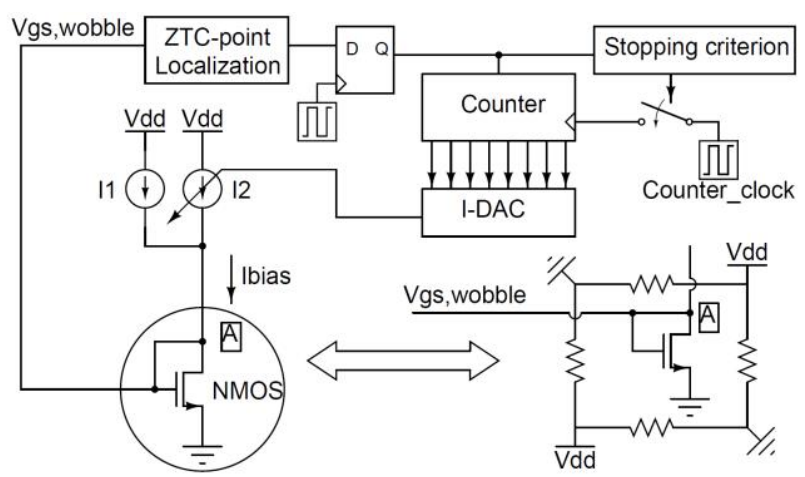

Fig. 2 Block schematic of the regulation system. The NMOS transistor (A) is surrounded by heating resistors. The 8-bit counter acts as a digital integrator.

Application in an oscillator circuit: The oscillator circuit has first been published by P. Geraedts on ISSCC 2008 for its outstanding phase noise behaviour. The operating principle is discussed elaborately in [4]. A simplified schematic of the oscillator is shown in fig. 3. The oscillation frequency is given by:

$$
\mathrm{f}_{\text {osc }}=\frac{\mathrm{I}_{1}}{\mathrm{C}_{2} \times \mathrm{V}_{\text {ref, } 2}}
$$

where $\mathrm{V}_{\text {ref,2 }}$ is equal to the gate voltage of M1 when the current through M1 is equal to $I_{1}+I_{2}$. At that moment the gate and drain of this transistor are at the same voltage. This current to voltage ratio strongly depends on temperature unless M1 is biased in its ZTC biasing point. Since the size of the capacitor as well as the charging current and reference voltage can be made temperature independent, the oscillator frequency will be temperature stable. Note that a replica of M1 is used in the calibration circuit in order to be able to do the calibration during circuit operation.

The simulation results are obtained for an oscillator which is designed to be ZTC biased in the typical process corner of a $130 \mathrm{~nm}$ technology. In this corner the frequency deviation from $0^{\circ} \mathrm{C}$ to $100^{\circ} \mathrm{C}$ is around $0.2 \%$. However, when simulating the same uncalibrated circuit in other process corners the frequency deviation over temperature increases up to $3 \%$. By using the calibration circuit this deviation can be diminished down to $0.4 \%$. Note that this technique can also be used to reduce supply voltage dependency since the ZTC point is unique for each MOS transistor and independent of external parameters. Also statistical 
transistor variations can be countered using the wobble-calibration technique. In applications where power consumption is an issue, one can switch off the calibration circuit once the ZTC point is reached.

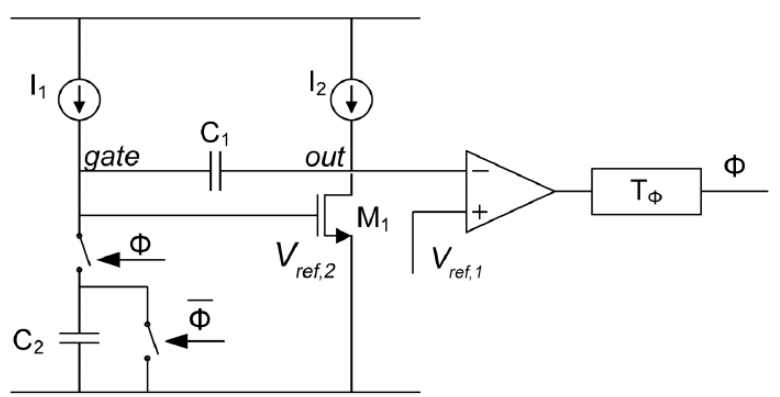

Fig. 3 Schematic of the switched capacitor oscillator presented in [5] and used as example circuit.

Conclusion: This letter has introduced a fully-integrated automatic calibration technique for temperature-independent, high-performance circuits. Using periodical heating of a MOS transistor the circuit can be biased in its ZTC point. The presented technique can be used in many applications going from temperature-independent timing circuits to temperature and supply voltage independent reference voltages or currents for DACs and ADCs. The calibration technique has been proven using finite-element temperature simulations combined with circuit simulations of an existing oscillator topology. The temperature sensitivity of the VCO has been reduced from $3 \%$ to $0.4 \%$ over all process corners.

V. De Smedt, W. Steyaert, W. Dehaene and G. Gielen (ESAT-MICAS, Katholieke Universiteit Leuven, Kasteelpark Arenberg 10, 3001 Heverlee, Belgium)

E-mail: valentijn.desmedt@esat.kuleuven.be

\section{References}

1 De Smedt, V.; De Wit, P.; Vereecken, W.; Steyaert, M.; , "A fullyintegrated Wienbridge topology for ultra-low-power $86 \mathrm{ppm} /{ }^{\circ} \mathrm{C} 65 \mathrm{~nm}$ CMOS $6 \mathrm{MHz}$ clock reference with amplitude regulation," Solid-State Circuits Conference, 2008. ESSCIRC 2008. 34th European, vol., no., pp.394-397, 15-19 Sept. 2008

2 De Smedt, V.; Dehaene, W.; Gielen, G.; , "A 0.4-1.4V 24MHz fully integrated $33 \mu \mathrm{W}, 104 \mathrm{ppm} / \mathrm{V}$ supply-independent oscillator for RFIDs," ESSCIRC, 2009. ESSCIRC '09. Proceedings of , vol., no., pp.396-399, 14-18 Sept. 2009

3 Filanovsky, I.M.; Su Tarn Lim; , "Interaction of threshold voltage and mobility temperature dependencies applied to stabilization of current and voltage," Circuits and Systems, 2000. Proceedings of the 43rd IEEE Midwest Symposium on , vol.3, no., pp.1022-1025 vol.3, 2000

4 Makinwa, K.A.A.; Snoeij, M.F.; , "A CMOS Temperature-toFrequency Converter With an Inaccuracy of Less Than $\pm 0.5^{\circ} \mathrm{C}(3 \sigma)$ From $-40^{\circ} \mathrm{C}$ to $105^{\circ} \mathrm{C}$ "Solid-State Circuits, IEEE Journal of , vol.41, no.12, pp.2992-2997, Dec. 2006

5 Geraedts, P.F.J.; van Tuijl, E.; Klumperink, E.A.M.; Wienk, G.J.M.; Nauta, B.; , "A $90 \mu \mathrm{W} 12 \mathrm{MHz}$ Relaxation Oscillator with a $-162 \mathrm{~dB}$ FOM," Solid-State Circuits Conference, 2008. ISSCC 2008. Digest of Technical Papers. IEEE International, vol., no., pp.348-618, 3-7 Feb. 2008 